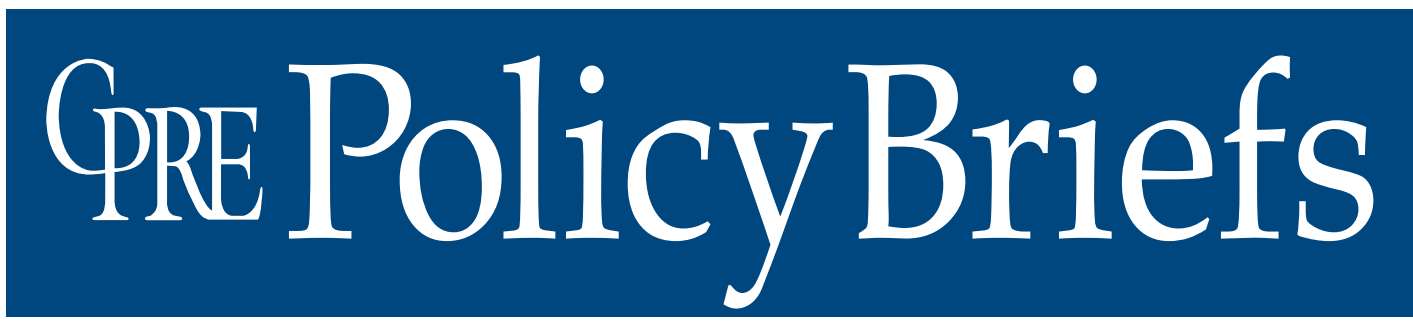

Reporting on Issues and Research in Education Policy and Finance

\section{Scaling Up Instructional Improvement Through Teacher Professional Development: Insights From the Local Systemic Change Initiative}

\author{
By Iris R. Weiss and Joan D. Pasley
}

There is a widespread view in the research and policy communities that the quality of mathematics and science instruction offered to students in the United States is low (Business-Higher Education Forum, 2005; Gonzales et al., 2004; National Commission on Mathematics and Science Teaching for the $21^{\text {st }}$ Century, 2000; National Research Council, 2000, 2001, 2005; National Science Board, 2004; Weiss, Pasley, Smith, Banilower, \& Heck, 2003). The widely discussed results of the Trends in International Mathematics and Science Study (TIMSS) suggested that the reasons for American students' poor performance in mathematics and science are complex, but at least partly due to weaknesses in the knowledge and skills of those teaching the subjects (Schmidt et al., 2001). In order to enhance teaching in these content areas, states and school districts need to act on what research has discovered about professional development (PD) and instructional improvement. The purpose of this brief is to share results from a major PD effort that extend our understanding of what is entailed in creating improvements at scale.

\section{The Consensus View}

A review of research over the last decade shows that typical PD, limited to a few in-service days a year, is ineffective in enhancing teaching (Corcoran, 1995). Elmore (2002) urges that in order to produce sustained gains in student learning, adequate opportunities for teachers to enhance both their pedagogical skill and content knowledge must be provided. Yet in spite of such recommendations and the pressures emanating from higher-stakes accountability systems, most PD opportunities remain fragmented, poorly aligned with curricula, and inadequate to meet teachers' needs - particularly acute in mathemat- ics and science- - for deeper knowledge of subject matter and understanding of pedagogy (Cohen \& Hill, 2001; Corcoran \& McDiarmid, 2000). Often the content of existing PD programs is unconnected to teachers' daily work, and little follow-up support is offered.

In recent years, researchers have begun to examine what works in PD. Supovitz, Mayer, and Kahle (2000) studied the effects of intensive, standards-based PD on science teachers in Ohio. They found that teachers became more positive about instructional reforms and more likely to use inquiry-centered pedagogy as a result of participating in intensive, standards-based PD. Cohen and Hill (2001) studied mathematics teachers participating in intensive curriculum-based PD offered by California in the 1990s. They found that participants were much more likely to make changes in practice than teachers receiving more general PD; these changes were associated with student performance gains. They concluded that providing teachers with extended, content-specific opportunities combined with follow-up support produced results. These findings are consistent with those of other studies, including a secondary analysis of the National Science Foundation (NSF) project that is the subject of this brief (Supovitz \& Turner, 2000). The general conclusion is that extended PD opportunities aligned with curricular content and accompanied by onsite follow-up support can produce significant changes in classroom practice and benefits for students.

On the basis of such studies, researchers and PD designers have reached broad agreement on key features of effective PD programs that lead to changes in teaching practices. (Elmore, 2002; Loucks-Horsley, Love, Stiles, Mundry, \& Hewson., 2003; Wilson \& Berne, 1999). According to
March 2006 RB-44

Graduate School of Education

University of

Pennsylvania

\section{Consortium for Policy Research in Education}

University of Pennsylvania

Harvard University

Stanford University

University of Michigan

University of Wisconsin-Madison 
this consensus view, high-quality PD programs are grounded in research and clinical knowledge of teaching and learning. They are aligned with a school's curriculum and assessments and focused on student learning in that setting. They facilitate teachers' collaboration both within and across schools, they use existing teacher expertise to plan activities and cultivate leaders, and they include mechanisms for garnering principal support. High-quality PD programs both model and explicitly discuss methods of good practice (such as inquiry-based methods in science) and provide teachers with active learning opportunities. These programs aim to build teachers' content knowledge and pedagogical skills. Finally, they are intensive, sustained over time to allow for integration of new knowledge into practice, and include follow-up support. While PD designed according to these principles does not guarantee that participating teachers will use what they learn to change their practice, it increases the likelihood that they will.

\section{The Local Systemic Change Initiative}

One major effort to provide teachers with such sustained, content-specific PD was instituted with NSF support in 1995. NSF initiated its Local Systemic Change through Teacher Enhancement (LSC) program in 1995 in order to improve instruction in science, mathematics, and technology through teacher PD. NSF funded the first cohort of LSC projects in 1995, and a new cohort of projects was added each year. By 2002, a total of 88 projects across the country had received LSC funding, typically for a period of five years each, with some projects using the first year for planning. By 2005, the LSC initiative had reached 70,000 elementary and secondary teachers (predominantly K-8) and two million students in 4,000 schools. The schools and students served by the LSC initiative included a high proportion of historically underrepresented groups. Nearly half of the schools targeted by the LSC were in urban areas, a quarter in suburban areas, and the rest about equally split between rural areas and towns or small cities. Across all schools targeted by the LSCs, just over half of the students were from minority groups.

The initiative aimed to involve all of a jurisdiction's teachers of the targeted subjects in particular grade bands in project-designated PD. Each targeted teacher was to participate in a minimum of 130 hours of PD over the course of the project. PD activities aimed to prepare teachers to implement high-quality mathematics and science
The Consortium for Policy Research in Education (CPRE) is funded by the Institute of Education Sciences, United States Department of Education under Grant No. R308A960003. Opinions expressed in this Brief are those of the authors and do not necessarily reflect the views of the Institute of Education Sciences, the United States Department of Education, CPRE, or its institutional members. This publication is based upon work supported by the National Science Foundation under contract numbers RED-9255369 and REC-9912485. Any opinions, findings and conclusions or recommendations expressed in this material are those of the authors and do not necessarily reflect the views of the National Science Foundation.

materials in their classes and to use inquiry-based practices that the materials supported. The chief aim of the LSC program was to encourage largescale reform in teaching practice by providing high-quality PD to all teachers and by supporting institutionalization of PD systems featuring enhanced practices and use of high-quality materials.

The PD provided by LSC projects proceeded from a clear theory of action in accordance with the research described above. The theory held that providing teachers with opportunities to deepen their content and pedagogical knowledge in the context of high-quality instructional materials would result in better-prepared teachers. The theory also predicted that with ongoing support, these teachers would be more inclined to change their instruction in ways advocated by national standards and would have more capacity to do so. Improved instruction would in turn lead to higher student achievement. This theory of action entailed common principles of high-quality PD opportunities that could improve teaching, of which three were crucial: establishing a supportive PD culture; providing PD experiences grounded in a combination of content, pedagogy, and materials; and using expert providers.

To realize the first principle, the initiative promoted efforts beyond the PD activities themselves to build a supportive environment for improving mathematics and science instruction. First, LSCs were charged with building a shared vision of mathematics and science education, designing a strategic plan for engaging teachers in PD, and promoting active partnerships and commitments among an array of stakeholders. LSC projects were also expected to align policy and practice within targeted districts and to engage in a range of activities to support reform. These included aligning mathematics and science curriculum frameworks, teacher evaluation systems, and student assessments with the LSC reforms.

To realize the second principle, designs for PD in individual projects typically included summer 
institutes of a week or longer as well as schoolyear follow-up support, e.g., study groups, classroom demonstrations, and coaching. Whether centralized or school-based, the LSC designs varied widely according to local needs. For example, some projects provided a great deal of PD prior to the teachers' implementing the instructional materials for the first time, while others delayed PD until after teachers had used the materials in their classrooms. Some LSCs used a cohort approach, for example, targeting one third of the teachers at a time, while others targeted all teachers each year. Regardless of the specific design, most projects estimated that it would take a teacher three or more years to reach the 130-hour target.

Instruction in content tended to be at the level at which students were expected to learn. Nearly half of PD time was devoted to engaging teachers in mathematics or science inquiry, with teachers working through problems as students might, in groups facilitated by project PD providers. Usually, teachers experienced about half of their PD hours in a large group, while about $40 \%$ took place in groups of under 15 people, and $10 \%$ in individual work, e.g., with coaches. Projects tried to weave together PD in content, materials, and pedagogy; time spent in these three areas was fairly balanced. According to evaluators, LSC designs were particularly effective when they guided teachers through a sequence of PD activities from materials-based workshops to more advanced activities emphasizing students' conceptual understanding and other pedagogical issues. Unlike typical PD, LSC work endeavored to create a year-round support structure for teachers by offering coaching and ongoing learning communities. However, for reasons described below, projects did not usually succeed in creating such a structure.

LSC projects used a diverse group of PD providers to enact the third principle. Typically, projects tapped university and industry scientists and mathematicians to address participants' content needs in particular. University science and mathematics education faculty and others from outside school systems (such as museum personnel) delivered PD in content, pedagogy, and materials. Teacher leaders from within systems (both teachers on special assignment and regular classroom teachers) also played a significant role in PD delivery. The leaders served as school-level resources for follow-up PD and as liaisons with project staff. They collaborated on district PD design and problem solving. Leaders on special assignment had more responsibility for planning and implementing PD and for providing coaching. District mathematics and science supervisors also led activities. Commonly, university faculty and teacher leaders worked together in teams, with teacher leaders focusing on pedagogy and materials, and university experts focusing on conceptual understanding.

In recruiting PD providers, projects sought individuals whose vision of mathematics and science instruction already matched that promoted by the LSC. At the same time, these providers represented a range of experience in content, teaching, and reform leadership. LSCs attempted to monitor providers, help them define their roles, and build their knowledge and skills. Regular staff meetings were a primary mechanism for doing so; one experienced project developed an active PD system for supporting providers. Projects that had worked for a number of years with PD providers-through the LSC and previously funded projects-often exhibited more highly developed strategies for provider support, demonstrating a key lesson learned from the initiative: that time matters.

How well did the experience of the LSC projects align with NSF's theory of action? Horizon Research, Inc., worked with project evaluators in a cross-site evaluation focused on the quality and extent of PD activities and their impact on teachers' attitudes and classroom practices in mathematics and science. Also addressed were questions about the degree of district and school support for the initiatives and about the extent of institutionalization of high-quality PD. Data gathered by evaluators of each project included observations of PD activities and classroom practices, teacher and principal questionnaires, interviews with teachers and principal investigators (PIs), and project ratings and project strategies questionnaires completed by PIs and LSC evaluators. Case studies and cross-site analyses were conducted as well. Banilower, Boyd, Pasley, and Weiss (2005) describe these measures and Horizon's data analysis methods.

This issue of CPRE Policy Briefs summarizes findings from the Horizon Research, Inc. evaluation. Broadly, three key insights emerge from the evaluation of the LSC effort. First, large-scale reform to enhance teaching should be carefully and specifically designed. Second, given such a design, system-wide PD can have significant impacts on instruction. Third, support across the 
system facilitates effective implementation of such PD. The brief examines these insights and also underscores the key challenges and successes of the LSC initiative. It shows what the design and implementation of this ambitious attempt to apply the consensus view of effective PD to mathematics and science accomplished.

\section{Design}

Large-scale PD reform must be carefully designed with particular features. While LSC designs were complex and varied considerably across projects, they contained common elements relevant to their impact.

\section{Goals}

Crucially, the implementation of LSC designs suggests that it is critical to set high and specific goals for the reform. NSF succeeded in that regard insofar as it was expected that the PD in each LSC would extend to all targeted teachers, be sustained over time, focus on content knowledge and use of research-based materials, be delivered in large measure by teacher leaders, and result in changes in practice. As will be shown, not all of those goals were attained perfectly, but the extent of attainment was impressive. These findings demonstrate that aiming high makes sense. Also, it helps to be prescriptive about goals while being flexible about implementation-this strategy proved to generate considerable creativity within individual LSCs.

At the same time, LSC project leaders found that the multitude of goals made project design difficult. For example, while they wanted a deep focus on developing teacher knowledge, they also wanted broad exposure to student instructional materials. Project staff were not always sure how to prioritize those goals. Moreover, designers wanted to counter the prevalent reform practice of encouraging teachers to develop/adapt instructional materials, but they did not make this aim salient, and observers noted that teacher adaptations often led to decreased opportunities for student learning. Overall, the LSC experience indicated that it is critical to begin an initiative by making primary —and achievable—goals clear to all involved.

\section{Prior Knowledge}

An important feature of the design of the LSCs was that they built on prior knowledge of what works in PD for science and mathematics teachers. The LSCs used an inquiry-based approach to instruction and materials designed to support the approach, which has been demonstrated by NSF studies and other research to benefit teacher knowledge and practice (for example, Bransford \& Donovan, 2005; Corcoran, 2003; National Research Council, 1999; Wiske, 1998). Built into the design as well was prior knowledge about the principles of effective PD, as described above. Particularly important was research demonstrating the importance of using existing expertise in providing $\mathrm{PD}$, gaining systemic support, and giving teachers opportunities to actively build knowledge. While use of prior knowledge did not always translate into success, it provided a framework to focus the work on the improvement of teaching and learning.

\section{Using Prior Knowledge}

The LSC design was also characterized by mechanisms for using the knowledge of what works-particularly mechanisms for partnerships, changes in policy, and capacity building. LSCs involved major partners as stakeholders, including universities, research institutes, and businesses. The most beneficial type of partnership was collaboration with a powerful external partner that could provide expertise, resources, and a continuing presence for reform. As one K-8 science evaluator said about an LSC's partnership with a university:

An important outcome of the project has been the development of the "partnership" among the five districts and the university. ... Each partner has brought considerable local strengths to the table to share with the others; each has been willing to explore new ideas presented and to reexamine local practices that stood in the way of achieving their goals. As the grant ends, there is every indication that this partnership will endure. (Banilower et al., 2005, p. 71)

Partnerships could not accomplish LSC goals alone; they had to be accompanied by plans for institutionalizing policy change that could mitigate factors in local contexts that threatened continuation of the work. NSF required proposers to have a plan for developing a supportive context for reform. Policies supportive of projects were indeed put in place, providing guidance and incentives and drawing attention to targeted aspects of teaching and learning. Overall, LSC districts were much more likely to have supportive policies in place in their last year than in their 
first (Horizon Research, Inc., 2005). Formal adoption of the project instructional materials and aligned curricular frameworks were perhaps most critical to this accomplishment.

Another mechanism of the design was capacity-building strategies. Over time, both NSF and project leaders realized the need for stronger efforts to build capacity by developing a cadre of teacher leaders who had effectively used project materials and could encourage such use among other teachers. Projects that were able to prepare teacher leaders adequately left an important legacy in their districts. Also important to capacity building was garnering support from principals for the work of teacher leaders. An important lesson was that project designs needed strategies for building capacity within the system, not just imported expertise and resources.

\section{Evaluation}

As a further design feature, NSF recognized the importance of program evaluation that would provide information helpful for mid-course project adjustments and that could be aggregated across projects. Ongoing evaluation work proved helpful for building a shared understanding of the vision for instructional change. Classroom observation protocols developed for the evaluation helped a variety of users to better understand the elements of high-quality teaching and to look beyond surface changes to examine the accuracy of classroom content and the extent of student conceptual learning. Savvy project staff were able to use data collection instruments and training materials to create change in PD providers, for instance helping teacher leaders understand the instructional vision and getting district leaders to focus on policy alignment. The LSC experience argues for using data gathered during a program to inform ongoing reform and to make sure that programs have the capacity to disseminate such data effectively. Related to including evaluation in the design is establishing a community of learners among funders, project personnel, and evaluators. When means-formal meetings and informal networks - exist for collaboration of project leaders, information needed to improve design can be shared and taken back to project sites. NSF encouraged the development of such communities of learners through PI meetings and an electronic communication system, and these improved both PD efforts and the evolution of NSF funding strategies, prescriptiveness, and evaluation.

\section{Time}

Finally, a specific feature of the LSC design was the expectation that reform would take time and require support for continued improvement. As the length of the grants, the expectation for large numbers of PD hours, the ongoing evaluations, and the encouragement of systemic support suggest, NSF expected that the work of the LSCs would take place over a long period and that to achieve impact, the enhancement efforts would have to work their way through the system. In this regard, the design was aligned with the consensus view of effective PD.

The LSCs did indeed design for sustained reform, but were they effective? Evaluation results suggest that they were, with important limitations.

\section{Impact}

The LSC initiative shows that well-designed, systemwide PD can have significant impact on teachers, teaching practice, and possibly on students. Although the initiative did not reach its very ambitious goal of providing all targeted teachers with 130 hours of PD, in part because of teacher turnover, setting such a goal resulted in more "press" to go beyond volunteers, and the projects reached larger proportions of district mathematics/science teachers than did many previous PD initiatives. The quality of PD was variable. While many sessions were excellent, evaluators also noted many missed opportunities for deepening teacher understanding of content and pedagogical strategies; in general, PD quality suffered to some degree from ineffective delivery by teacher leaders. Thus the quality across projects was not as high as the ambitious designs, based on the consensus view, intended. Nevertheless the initiative clearly enhanced teaching in a number of ways.

\section{Attitudes and Perceptions}

One finding of the LSC evaluation data was that teachers had more positive attitudes towards reform-oriented mathematics and science teaching. For various grade-level and subject groupings (K-8 science, K-8 mathematics, and 6-12 mathematics), teachers' positive attitudes were correlated with the extent of participation in LSC PD activities. Evaluators indicated that teachers left PD activities with greater enthusiasm for teaching, heightened awareness of how students learn, and willingness to modify teaching practices and 
collaborate with colleagues. Teachers attributed new beliefs about how students learn-through questioning, discovery, and reflection-to their exposure to inquiry-based instruction in LSC project PD. Evaluators also noted changes in some teachers' beliefs about the ability of all children to learn mathematics and science.

Participation in LSC PD activities appears also to have had a positive impact on teachers' perceptions of their pedagogical preparedness to teach mathematics and science. Across gradelevel and subject groupings, their sense of preparedness, as measured by questionnaire data, increased with increasing participation in project PD. Even when extent of participation was controlled for, the sense of preparedness increased as the projects matured, indicating a systemic effect on preparedness. Results were similar, and somewhat stronger, for teachers' perceptions of their content preparedness. Again, more participation meant a greater sense of preparedness, with interview data showing teachers who had participated in 60 or more hours of PD more likely to report an impact on content knowledge than teachers with fewer hours. However, these results must be viewed with caution; evaluators noted that some teachers might have overrated their preparedness, and others might have felt less prepared as they grew to understand the complexities of effective instruction.

\section{Mathematics/Science Content of Lessons}

Teachers' participation in LSC PD was linked to a number of positive outcomes in their instructional practices. One key finding was that participation was connected with higher ratings of the quality of mathematics and science content in observed lessons. Teachers with greater participation were more likely to present significant and developmentally appropriate content and to present the subjects as a dynamic body of knowledge. Teachers grew in content knowledge over time participating in $\mathrm{PD}$, as one evaluator of a K-8 mathematics LSC explained:

A deeper understanding of the content is reflected in the teacher-student interactions observed in classrooms. Teachers are better able to ask questions that have the potential for leading to greater conceptual understanding among the students. This would suggest greater understanding of the mathematics content. (Banilower et al., 2005, p. 47)
However, teachers' capacity to address content ranged widely, with evaluators citing evidence of teacher misconceptions and difficulty translating knowledge into student learning.

\section{Pedagogy of Lessons}

Another key finding was that participation in LSC PD was linked to increased use of reformbased pedagogy, including use of hands-on activities, extended investigations, and work on models/simulations. Over half of teachers participating in the PD indicated that participation had influenced their selection of instructional strategies; those engaging in 60 or more hours of treatment were more likely to report this impact. Analyses of questionnaire data showed a positive, nonlinear relationship between PD participation and a composite measuring reported frequency of use of the investigative practices encouraged by the reform, with most of the impact occurring within the first 80 treatment hours, as shown in Figure $1{ }^{2}$ However, the increased confidence that teachers frequently reported in their ability to teach the subjects was sometimes misplaced, with observers noting lower levels of appropriate pedagogy than self-reports would suggest. Such discrepancy between self-reports on preparedness and actual teaching behaviors may stem from teachers' limited understanding of the depth of change that LSCs sought.

The findings on pedagogy were thus decidedly mixed. Observations early and late in the life of LSCs did support the notion that teachers' use of instructional practices aligned with the LSC vision grew over time as a result of LSC PD, with teachers more often using investigative practices, questioning students effectively, and helping them make sense of lesson content. The extent of PD participation and use of LSC materials were both positively correlated with evaluators' ratings of the quality of teachers' questioning and sensemaking strategies. The data also show, however, that fewer than half of lessons, even those of the teachers with the greatest PD participation, were rated highly in these two areas. Observations fre-

\footnotetext{
This composite (and others presented in this brief) was calculated as the percentage of total possible points on a group of questionnaire items related to a single construct.

"The figures in this brief show "predicted" values of an outcome. These predicted values are calculated from the regression equation resulting from the analysis, which controlled for a number of teacher, school, and/or project characteristics. Thus these values represent the outcome for a "typical” LSC teacher at various levels of PD or a "typical" LSC project at various times from receiving funding.
} 
Figure 1. Predicted Composite Scores: Use of Investigative Practices

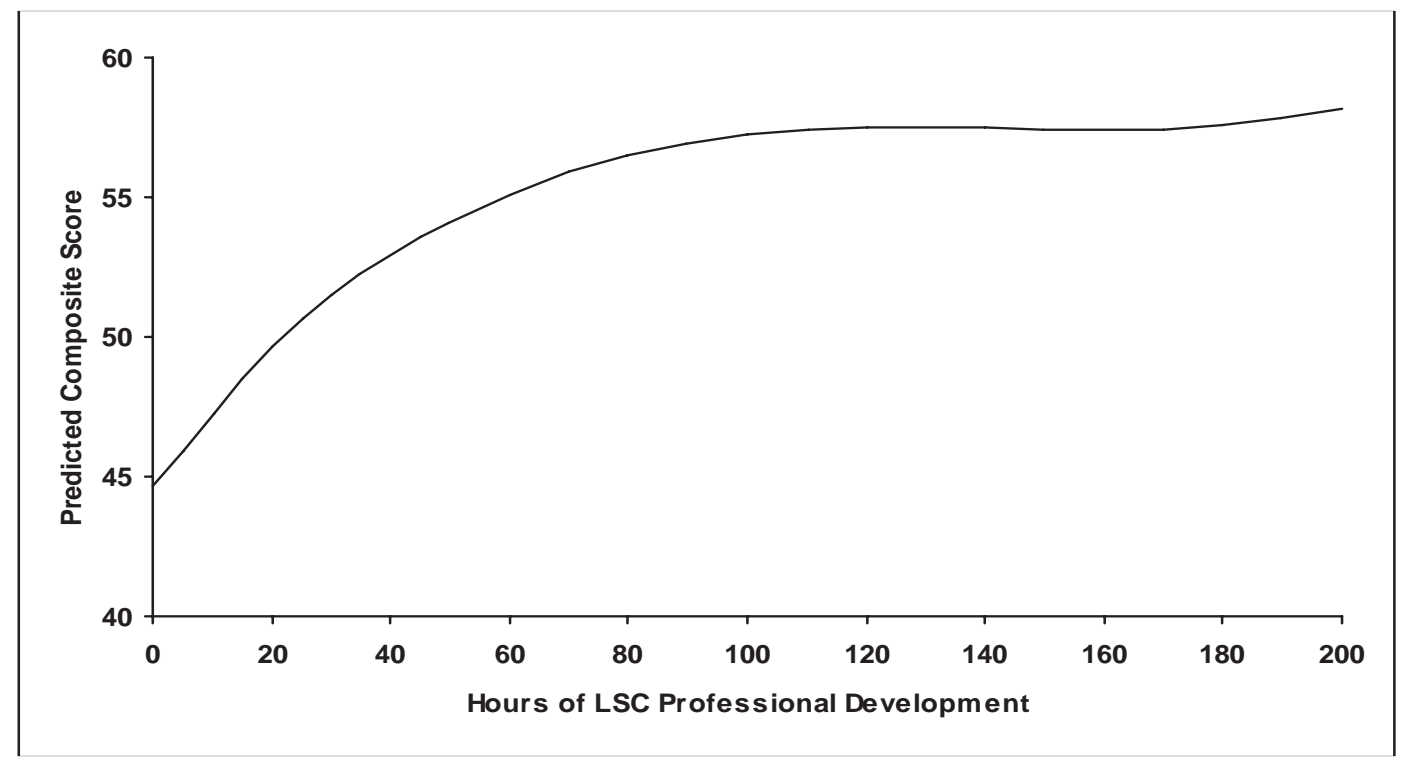

quently revealed both rudimentary and accomplished examples of mathematics and science instruction among LSC teachers. Many teachers were able to implement pieces of the reform, but few could deliver all the components to students. Much of the change in instruction cited by teachers struck evaluators as superficial. Case studies revealed pedagogical weaknesses in LSC teachers, such as limited student engagement with concepts in modules, mechanical implementation of instructional materials, and infrequent use of sophisticated questioning and sense-making strategies. Overall, LSC efforts succeeded in shifting teachers' thinking around pedagogy, but the program had more limited impact on teachers' ability to fully operationalize their learning in this area.

\section{Materials Used in Lessons}

Also important was the finding that the use of district-designated instructional materials was positively correlated with more reform-based content and pedagogy. In interviews and on questionnaires, teachers who participated in a considerable amount of LSC PD reported more frequent use of the materials than did teachers with fewer hours. As with other outcomes, most of the change occurred in the first 80 hours of PD. Analysis of these data also showed a systemic impact, with teachers' reported use of the designated materials increasing as projects matured, even after controlling for teacher participation in the PD.

While the use of instructional materials was fairly widespread in observed classrooms of LSC teachers, the quality of use varied tremendously.
Evaluators were trained to use an observation protocol that examined the design and implementation of lessons, the learning climate of the classroom, and the quality of the mathematics/science content in the lesson. Observers used this information to rate the extent to which the lesson embodied the principles of teaching for understanding (e.g., providing learning experiences aligned with developmentally appropriate learning goals, using high-order questioning strategies, and providing students opportunities for sensemaking). While some teachers did an excellent job of teaching for understanding, others conducted lessons that focused on procedural knowledge rather than on the deeper conceptual knowledge that the materials were designed to foster. Evaluators reported that teachers in the early stages of learning were more likely to use materials mechanically, or to modify them inappropriately. Some teachers moved to activities on advanced concepts without an adequate foundation. Still others omitted activities, revised lessons, or added only loosely related supplementary materials, thus hampering the development of student conceptual knowledge. These adaptations often reflected teachers' concerns about time constraints, classroom management, and/or high-stakes testing. Teachers' lack of content knowledge also seemed to limit appropriate use of the materials; while many teachers engaged students with the materials, the lessons had little questioning and discussion to promote concept development. Some teachers came to perceive these shortcomings over time. As one K-8 science evaluator said: 
One positive effect of the program is that teachers recognize the difference between hands-on activity in the science classroom and authentic, rigorous investigation. They realize that they are becoming more capable in the hands-on part of science exploration, but they also recognize that they are less fluent with the minds-on part of exploration. (Banilower et al., 2005, p. 55)

Evaluators did observe noticeable classroom improvements attributable to implementation of the materials. Overall, while use of the materials was far from perfect, evaluation data suggest that it enhanced the quality of instruction.

\section{Classroom Culture}

The evaluation revealed that LSC projects had an impact on the culture of mathematics and science classrooms. Analysis of teacher questionnaire data found that time spent in LSC PD was associated with higher scores on a composite measure of practices that foster an investigative classroom culture, such as arranging classroom seating to facilitate student discussion, requiring students to supply evidence to support their claims, and encouraging students to consider alternative explanations. The typical participant scored 7.69 points higher (effect size 0.38 standard deviations) than the typical nonparticipant, with most of the gain occurring in the first 80 hours of PD. Evaluator ratings of observed lessons support this finding. Lessons were more likely to be rated highly for actively involving all students, engaging students intellectually, and creating a climate of respect and rigor when taught by teachers with high levels of LSC PD.

Nevertheless, evaluators also observed much classroom practice that remained far from investigative. In one class, a teacher set the stage for inquiry by encouraging students to discuss what they already knew about the concept of melting. But when students showed misunderstanding of the concept, the teacher abandoned questioning strategies and resorted to merely telling students what melting was, thus relapsing into familiar teaching behaviors that precluded further investigation. Evaluators suggested that factors such as lack of content knowledge, lack of confidence with the investigative model and materials, and resistance to change contributed to such limitations on teachers' capacity to create an investigative classroom culture.

\section{Instructional Quality}

Evaluator ratings of instructional quality support the findings on attitudes and practices. The extent of teacher participation in LSC PD and the use of the LSC materials were positively associated with higher evaluator ratings of lesson quality, and the combination of PD and materials use had a greater effect than either alone. Lessons taught by teachers with at least 80 hours of PD and based on the materials were more than twice as likely to receive a high rating as lessons of untreated teachers not using the materials. An analysis of teacher observation data that accounted for the mediating effects of teacher attitudes, preparedness, and principal support found similar associations. Some differences were evident with lower levels of participation in LSC PD; lessons taught by teachers who had participated in at least 20 hours of LSC PD were more likely to be judged strong in a number of areas, such as the accuracy and significance of content, the climate of respect for student contributions, the engagement of students with important ideas, the likelihood of teachers' questioning strategies enhancing conceptual understanding, the valuing of intellectual rigor and constructive criticism, and the match between conceptual understanding and students' developmental level.

The LSC program also had an effect on the amount of time spent on science teaching in selfcontained classes in the elementary grades. For example, Heck and Crawford (2004) found a positive relationship between the extent of teachers' participation in LSC PD and the minutes per week that elementary teachers spent on science instruction (see Figure 2). The effect size was moderate, equivalent to 6.21 minutes a week. Teacher participation in LSC PD fully explained this increase in instructional time as projects matured. There was no systemic effect for nonparticipating teachers in LSC districts.

\section{Student Impact}

Data on the direct impact of LSCs on students is quite limited at this time. While some projects included a design for assessing student impact, NSF did not require LSCs funded in the program's first four years to examine this outcome. Of the 58 projects funded during this time, 16 provided results from student impact studies. Later cohorts were required to study student impact, but many of these projects have not yet completed their studies. Reviews of the 16 studies, a Horizon Research, Inc. three-year, cross-site study of LSC 
Figure 2. Time Devoted to Science Instruction in Elementary

Science Classes

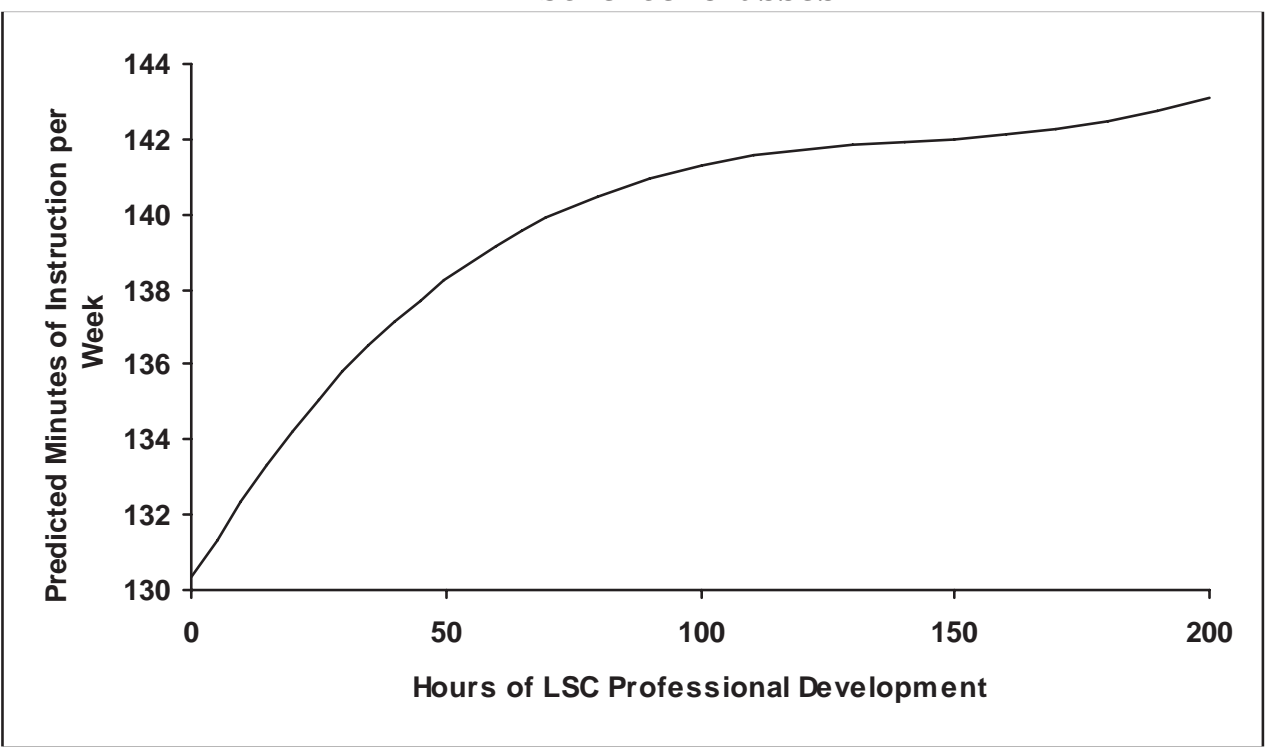

impact on elementary science achievement, teacher interview data, and principal questionnaire data provide some information on student impact. The reviews (Banilower, 2000; Zhang \& Wang, 2002) revealed evidence of positive impact on student achievement, attitudes, and course-taking patterns in both mathematics and science LSCs. For example, in one LSC, Amaral, Garrison, and Klentschy (2002) found a positive correlation between student achievement in both science and writing proficiency and the number of years a student received instruction from LSCtrained teachers using district-designated instructional materials. However, constraints on research design in many of the studies, such as lack of a comparison group, made it difficult to judge whether the findings were attributable to the LSC.

Data from the first two years of a cross-site science program study also showed some positive student impact. The study used a quasi-experimental, pre-test/post-test design to determine how teacher participation in LSC PD affected student achievement. First-year results indicated that the extent of teacher participation in LSC PD was a significant, positive predictor of student achievement, after controlling for prior achievement and demographics. However, problems in data collection prevented the analysis from including the amount of science instruction received or the extent of use of LSC instructional materials.

Second-year results showed that teacher participation in LSC PD was a positive predictor of the amount of science instruction and the use of the instructional materials. Yet neither factor was a significant predictor of student achievement. Moreover, projects in the study were typically unable to require teachers to participate, so the possibility of selection bias was a concern. Finally, teacher interviews and principal questionnaires indicated a positive correlation between involvement in PD and reports of the impact of LSCs on student and school achievement. The available data thus suggest that the LSC program has had a positive impact on students. Forthcoming data from projects required to examine student impact should shed additional light on the extent of that impact.

In summary, LSC PD had widespread impact on teaching and possibly on students, but the impact was not consistent and often not very deep. At the same time, challenges to consistent and deep implementation of investigative practices through LSC PD and materials were somewhat mitigated by an important adjunct to LSC core activities, the development of systemic support.

\section{Systemic Support}

NSF expected LSCs to build support throughout the system for comprehensive implementation of the PD design. Projects attempted to build support in different ways. Important strategies were alignment of policies with the project vision, developing supportive structures and capacity for $\mathrm{PD}$, and developing administrative support as well as external partnerships. Where system support was present, implementation of the LSC vision of 
Figure 3. District Systems Composites

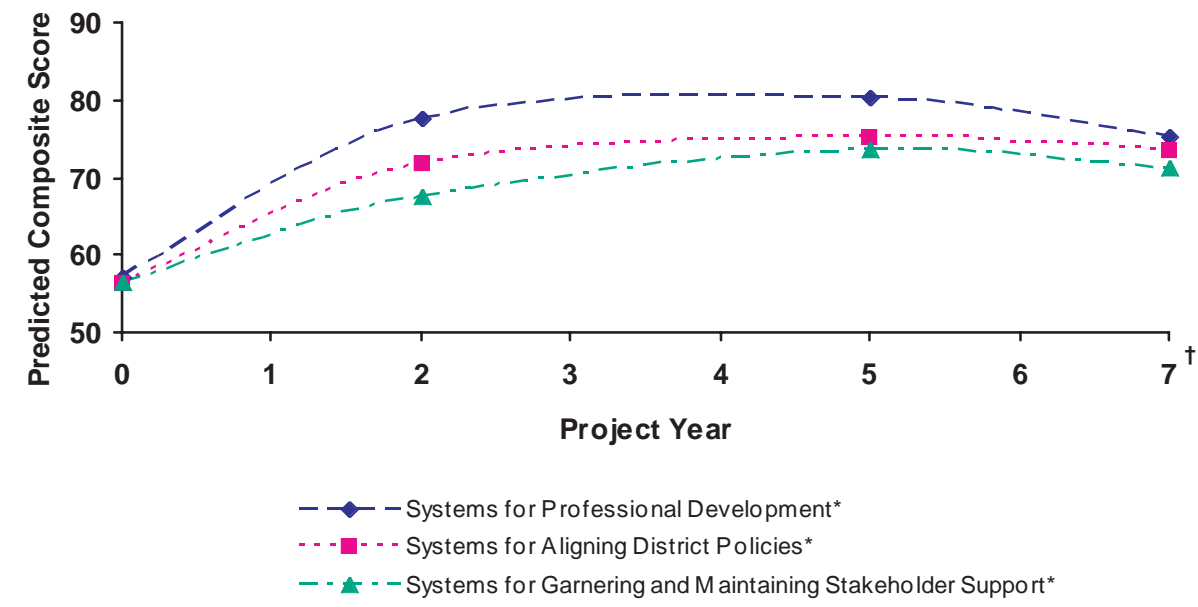

Project Year Seven data are predictions about future systems made by the project during its final year.

Composite score changes significantly over time, $\mathrm{p}<0.05$.

instruction seemed more effective and more likely to be sustained.

\section{Alignment}

The evaluation found that despite the fact that the policy context in which the LSCs operated was characterized by much administrative change and conflicting priorities, many LSCs were successful in aligning curriculum frameworks, assessments, and teacher evaluations with the LSC vision for mathematics and science instruction, and in securing widespread adoption of exemplary materials by schools and districts. These successes fostered systemic changes in classroom practice, with both participating and nonparticipating teachers using practices and materials aligned with LSC principles. District leaders praised the alignment that LSCs promoted. One K-8 science administrator said that after the LSC work,

Everyone understands the science agenda and is making sure schools are paying attention to it ... We think of ourselves as a coordinated system, serving the schools ... and that is a whole different way that this system has aligned itself. (Banilower et al., 2005, p. 73)

The alignment of district policies and LSC principles took time; evaluators found that districts began with relatively weak systems for alignment with LSC reform, but alignment increased over the first two project years and was maintained to the end (see Figure 3). Riding the wave of standards-based reform in the 1990s,
LSCs played critical roles in promoting both awareness and implementation of aligned policies throughout systems, particularly coordinated use of standards-based instruction and exemplary materials. Nonetheless, conflicting priorities sometimes interfered with alignment, which varied widely across projects.

\section{Structures}

Many LSC districts developed other structures that supported the LSC vision of PD systems for mathematics and science. Evaluators cited numerous ways in which LSCs influenced districts to support PD. In one urban district, more strategic planning and coordination across central office departments resulted in stronger support for sustained PD for teachers and principals. Some LSCs successfully pushed for mandatory PD. Among the most important structures initiated by LSCs were those to ensure PD for teachers new to the instructional materials. In one LSC, five districts jointly established a beginning teacher program to ensure training for newly hired teachers. Many systems had LSC-initiated PD structures, such as summer training, that remained in place after project funding ended. Evaluators deemed the most important legacy of the LSCs to be the expertise of teacher leaders. A number of LSC projects provided strong, ongoing support for teacher leaders, and continued to support these leaders in PD roles, such as mathematics coaches, after projects ended. However, LSCs sometimes underestimated what was needed to develop and sustain school-based PD, and overestimated the capacity of project staff and lead teachers to do 
this work. Teacher leaders progressed at a rate slower than anticipated by LSC staff-evidence of a time lag between capacity-building efforts and teachers' ability to make gains in competence. This finding shows again that effective PD needs support over time to succeed.

\section{Administrative Support}

Support from school and district administrators was crucial to the success of LSCs. LSC PIs typically cited principal support as the most important factor in determining teacher participation in PD and in developing a supportive context for reform in schools. Both PIs and principals themselves noticed deeper commitment to PD as a result of LSC initiatives, and schools without principal turnover were significantly more likely to have positive outcomes on several measures of movement of LSC activities and materials into schools (Crawford \& Banilower, 2004). On some measures, LSCs had diminished impact in schools with principal turnover. Overall, principal support was important but proved challenging for LSCs, with many principals paying lip service to reform without setting expectations for PD participation or materials use.

On the other hand, efforts to gain the support of district administrators were less problematic. Where LSCs established strong working relationships with superintendents, school boards, curriculum directors, and others, the potential for sustained support increased significantly. In one multidistrict LSC, superintendents were engaged as co-PIs, and in turn the superintendents helped remove roadblocks to $\mathrm{PD}$ participation and materials adoption. Across LSC projects, district leaders often demonstrated their support by allocating funds for replenishing science materials and providing resources for training new teachers. Midlevel district administrators often proved the strongest and most stable allies; with low turnover, they could become a loyal group engaged in promoting the LSC vision and aligning LSC reforms with district priorities. However, high superintendent turnover and competing priorities, such as the need to align materials with state assessments, did cause difficulty in sustaining district support.

\section{Partnerships}

As indicated earlier, LSCs were designed to involve external partners. The most effective external partners (e.g., universities, museums) provided not only resources but also help in establishing stable structures for sustaining reform, such as centers for disseminating materials and PD. Access to expertise of university mathematics and science faculty also tended to be an advantage. Although attempts to build partnerships with universities and other assistance providers were limited by time, resources, and the demands of the LSCs' primary mission of teacher enhancement, collaboration with partners sometimes resulted in support for LSC principles that lasted beyond the end of projects.

\section{Institutionalization}

LSCs aimed to institutionalize reforms beyond the end of NSF support. Given the rapidly changing education policy context, institutionalizing key elements of reform is essential for lasting impacts (Stone, Henig, Jones, \& Pierannunzi, 2001). Through garnering both internal and external support, building district and school capacity, and encouraging adoption of supportive policies, LSCs took many steps to institutionalize a PD system aligned with the LSC vision for teacher enhancement. However, evaluators found that there was not a significantly greater likelihood of institutionalization of reform components at Year Five of projects than at Year Two (see Table 1).

An erratic policy environment made institutionalization of PD activities and materials use difficult. Established support structures for PD were jeopardized by cuts in state and district funding, changes in state assessments incompatible with the LSC vision, and particularly in science, moves to eliminate emphasis on PD in areas other than literacy and mathematics. Other barriers to institutionalization arose from LSCs themselves. For example, PIs often had more expertise for implementing PD activities than for dealing with issues related to policy and systems. Despite some success in institutionalization, such as the policy changes discussed earlier, in general LSC designers' limited understanding of the scope of the work required for institutionalization limited their ability to translate LSC activities into systemic reform. Designers understood that institutionalization was crucial to extending the scale of its PD efforts after funding ended, and they grasped that institutionalization relies on a major commitment of resources over time. Yet they were not able to grapple effectively with the challenges to this understanding that the reality of LSC implementation posed.

Many internal and external challenges affected the success of LSCs. The most significant of these are described in the following section. 
Table 1. Continuum Ratings of Likelihood of Institutionalization of LSC Reforms

\begin{tabular}{lcc}
\hline & \multicolumn{2}{c}{ Percentage of Projects } \\
\hline Lear Two & $\begin{array}{l}\text { Final Year } \\
\text { (N=61) }\end{array}$ \\
\hline Level & 0 & 0 \\
Level 3: Minor Components Likely to B ecome Institutionalized & 30 & 19 \\
Level 4: Components Likely to Become Institutionalized & 63 & 62 \\
Level 5: Institutionalization of LSC Reforms Likely & 8 & 17 \\
\hline
\end{tabular}

\section{Challenges}

LSCs faced several sets of challenges that tended to limit the impact and staying power of reforms. A first set of crucial challenges arose in the training of PD providers. While LSCs were typically rated highly in the quality of their preparation of PD providers, evaluators also pointed to areas of weakness among providers that sometimes limited impact. These weaknesses were seen in both content sessions (e.g., lack of rigor) and pedagogy sessions (e.g., lack of explicit discussion of strategies). These limitations could be explained in part by the need for LSCs to press PD providers into service before they were fully prepared and in part by limited resources that constrained the time and effort devoted to preparing providers. To address this challenge, more sustained effort around provider preparation and support was needed, especially in areas that posed particular difficulties for teachers, such as unfamiliar content, higher-order questioning and lesson closure. Projects might have trained providers better around the mathematics and science concepts underlying the student modules, might have illustrated student-centered instruction and student interaction more clearly, and might have more effectively presented ways to close lessons by helping students make conceptual connections.

A related set of challenges for LSC PD was deepening teachers' understanding of mathematics and science content. In part, the emphasis that projects placed on materials and on the mechanics of the modules and kits distracted from broader content needs. In addition, some providers, particularly teacher leaders, seemed underprepared for helping teachers make sense of content. Moreover, PD sessions designed to deepen content knowledge and support teachers' content needs during implementation sometimes failed because other more pressing teacher concerns, such as materials management, dominated the sessions. Again, these difficulties might have been addressed through better preparation and support for the providers. In addition, projects might have devoted a more equitable share of PD time to content and have created more content-specific sessions matched to teachers' needs.

Further, supporting teachers during implementation posed challenges for LSCs. While projects typically provided teachers the supplies needed for the implementation of the modules and kits, they devoted far less attention to ongoing individual and small-group support, partly because of lack of resources and staffing. Teacher leaders, typically charged with providing ongoing support, were often spread thin and/or lacked the coaching and facilitation skills needed for engaging teachers effectively. Time constraints also led to difficulty in recruiting teachers for participation in the follow-up activities that were offered to build communities of learners and foster reflection. Moreover, lack of school support and formal structures limited follow-up opportunities during the LSCs and pointed to difficulties in sustaining support for teachers beyond the grant. These challenges suggest the need for cultivating greater awareness among district and school administrators, who could have encouraged teacher participation in ongoing PD activities and fostered opportunities for collegial interaction and mutual support.

Indeed, another set of challenges arose around engaging principals, who played key roles in determining the outcomes of LSCs-from 
encouraging teachers to participate in LSC activities, to enabling the work of teacher leaders, to making time for teachers to participate in ongoing site-based activities. Stable administrative leadership in schools was crucial, impacting the likelihood of teachers using LSC materials. LSCs struggled with balancing resources and effort devoted to teacher PD with reaching out to other stakeholders such as school leaders. Attention to principals was sometimes neglected altogether. Projects clearly had little control over some barriers affecting the extent of principal support, such as turnover, but they might have devised deliberate strategies for communicating their message to administrators through existing structures. Engaging principals in ongoing and fairly intensive ways would have been a worthy pursuit with potentially large payoffs both during and beyond the life of projects.

Finally, projects found it challenging to reach all the targeted teachers. Despite efforts to provide a range of opportunities, LSC projects were not able to engage most eligible teachers in 130 hours of PD. Teacher turnover, state and district policies that reduced time available for project $\mathrm{PD}$, and teacher reluctance to participate all contributed to this challenge. In some districts, the large influx of new teachers, as well as mobility across schools and subject areas, hindered efforts to meet the 130-hour goal. Many LSCs underestimated teacher turnover and were forced to improvise new strategies to meet the needs of new teachers. Projects might have better anticipated teacher turnover, making plans to include new teachers and to offer them incentives for participation. Beyond this, projects could have looked for more effective strategies to make the case for reform with both teachers and administrators, and continued to make the PD opportunities attractive even to those resistant to the reform vision.

\section{Successes}

Along with these challenges, the LSC initiative experienced major successes, as has been shown. First, core evaluation data suggest that projects have done well in providing teachers with high-quality PD around mathematics and science, the initiative's primary aim. Evaluators' ratings for overall quality of PD programs were high across several dimensions. In particular, LSCs were strong in creating a culture conducive to teacher learning, and in preparing teachers to use high-quality materials and appropriate pedagogy in their classrooms. Further, ratings for overall program quality and the quality of individual sessions improved significantly over time, suggesting that LSCs became more adept at implementing high-quality PD as they matured and reinforcing the consensus view on PD that time matters. Indeed, time spent in PD seems an essential component for scale-up of instructional reform in mathematics and science.

Success was also evident in the positive impact of LSC PD on teaching and learning. Teachers' attitudes toward reform-oriented teaching and their perceptions of their content and pedagogical preparedness improved with increased participation in LSC PD. Teachers' participation in LSC PD was linked to the positive instructional outcomes described above, such as enhanced quality of lesson content and more frequent use of investigative practices and materials. Evaluation data also suggest that the quality of classroom instruction improves over time as teachers' PD hours accrue, at least to the level of 80 hours. Although there were minor differences in impacts for mathematics and science teachers, overall, these results were equally evident in both subjects.

Moreover, the widespread use of high-quality mathematics and science materials in the classroom by participating teachers points to program success. LSC PD focused heavily on giving teachers opportunities to explore the materials through practice, investigation, problem solving, and discussion strategies that likely contributed to teachers' willingness to use the materials with their students. Evaluation data suggest that the quality of instruction improved with teachers' use of the designated instructional materials, and that teachers were more likely to use the materials as they accumulated LSC PD hours. The LSC initiative also worked to ensure that the materials became established by promoting their formal adoption in participating districts. These efforts showed a systemic effect, increasing use over time among both participating and nonparticipating teachers in LSC districts.

Finally, LSC projects had success in building stakeholder and policy support for the LSC vision and interventions. While LSCs began in an environment leaning toward reform, participating districts were less likely to have systems in place to support reforms before projects began. In contrast, districts were much more likely to have these systems in place in projects' last year, sug- 
gesting a link between project efforts to build a supportive context and the institutionalization of PD systems, as well as the development of stakeholder and policy support. The adoption of LSC materials and aligned frameworks, district support of teacher leaders in varying capacities, and the development of external partnerships all demonstrate the success LSCs have had in building capacity to support and sustain reform.

\section{Conclusion}

The LSC initiative has shown that PD to enhance mathematics and science teaching can be implemented effectively at scale. When carefully designed and energetically supported, ambitious PD focused on instructional content and materials and sustained over time can change what happens in classrooms; impacts on teachers and their teaching were typically evident after approximately 30 hours of PD, with further impacts detected through 80 hours of PD. The LSC evaluation suggests some implications of importance to those considering similar large-scale instructional reform through PD. First, if PD is going to have an impact, it needs to be focused on clear goals and delivered over time by well-trained providers. Competing and distracting PD efforts should be discouraged. Second, if PD is to move beyond business as usual, it must be based on content and practice and planned as a coherent set of strategies to develop teachers' content and pedagogical knowledge. This work is difficult, but it does not require starting from scratch; most districts have internal capacity — such as teachers who can lead PD and principals who can align instruction with a reform vision - that can be used to move instructional change to scale. Third, alignment of district policies with instructional reforms and garnering the support of school/district administrators is crucial to the success and long-term sustainability of these reforms. Finally, stakeholders need to be aware that change takes time-and work to help teachers gain that time for learning. The findings from the LSC program thus support the consensus view about effective PD: the importance of content-based PD, aligned with curriculum and assessment, focused on student learning, sustained over time, with collaboration among teachers, and administrative support. The LSC experience also points to some ways to make sure the common sense of that consensus becomes common in practice.

\section{About the Authors}

Iris R. Weiss is President of Horizon Research, Inc. Her current research focuses on the implementation and impact of large-scale reforms, including systemic initiatives, and the NSF Math and Science Partnerships. Previous research has included studies of the status of mathematics and science education, including a series of national surveys of science and mathematics teachers, and observations of a large, nationally representative sample of K-12 science and mathematics classes.

Joan D. Pasley, Senior Research Associate at Horizon Research, Inc., serves as the program manager of the standardized evaluation system for the Local Systemic Change (LSC) program. She also directs the evaluations of a number of NSF's Math and Science Partnerships.

\section{References}

Amaral, O. M., Garrison, L., \& Klentschy, M. (2002). Helping English learners increase achievement through inquiry-based science instruction. Bilingual Research Journal, 26(2), 213-239.

Banilower, E. R. (2000). Local systemic change through teacher enhancement: A summary of project efforts to examine the impact of the LSC on student achievement. Chapel Hill, NC: Horizon Research, Inc.

Banilower, E. R., Boyd, S. E., Pasley, J. D., \& Weiss, I. R. (2005, prepublication copy). Lessons from a decade of mathematics and science reform: A capstone report for the local systemic change through teacher enhancement initiative. Chapel Hill, NC: Horizon Research, Inc.

Bransford, J. D., \& Donovan, M. S. (2005). Science inquiry and how people learn. In M. S. Donovan \& J. D. Bransford (Eds.). How students learn: History, mathematics, and science in the classroom (pp. 397419). Washington, DC: National Academy Press.

Business-Higher Education Forum. (2005). A commitment to American's future: Responding to the crisis in mathematics and science education. Washington, DC: Author.

Cohen, D. K., \& Hill, H. C. (2001). Learning policy: When state education reform works. New Haven, CT: Yale University Press. 
Corcoran, T. B. (1995). Helping teachers teach well: Transforming professional development (CPRE Policy Brief No. RB-16). New Brunswick, NJ: Consortium for Policy Research in Education.

Corcoran, T. B. (2003). The Merck Institute for Science Education: A successful intermediary for school reform (CPRE Research Report No. RR-052). Philadelphia: Consortium for Policy Research in Education.

Corcoran, T. B., \& McDiarmid, W. (2000). Promoting the professional development of teachers. In R. Pankratz \& J. Petrosko (Eds.), All children can learn: Lessons from the Kentucky reform experience (pp. 141-158). San Francisco: Jossey-Bass.

Crawford, R. A., \& Banilower, E. R. (2004). LSC principal questionnaire study: An analysis of data collected between 1997 and 2003. Chapel Hill, NC: Horizon Research, Inc.

Elmore, R. F. (2002). Bridging the gap between standards and achievement: The imperative for professional development in education. Washington, DC: Albert Shanker Institute.

Gonzales, P., Guzman, J. C., Partelow, L., Pahlke, E., Jocelyn, L., Kastberg, D., et al. (2004). Highlights from the trends in international mathematics and science study (TIMSS) 2003 (NCES 2005-005). U. S. Department of Education, National Center for Education Statistics. Washington, DC: U. S. Government Printing Office.

Heck, D. J., \& Crawford, R. A. (2004). LSC teacher questionnaire study: Indicators of systemic change. Chapel Hill, NC: Horizon Research, Inc.

Horizon Research, Inc. (2005). Designing large scale education initiatives: Lessons from the Local Systemic Change Through Teacher Enhancement program. Chapel Hill, NC: Author.

Loucks-Horsley, S., Love, N., Stiles, K. E., Mundry, S., \& Hewson, P. W. (2003). Designing professional development for teachers of science and mathematics ( $2^{\text {nd }}$ ed.). Thousand Oaks, CA: Corwin Press.

National Commission on Mathematics and Science Teaching for the $21^{\text {st }}$ Century. (2000). Before it's too late: A report to the nation from the National Commission on Mathematics and Science Teaching for the $21^{\text {st }}$ Century. Jessup, MD: Education Publications Center.

National Research Council. (1999). How people learn: Brain, mind, experience, and school. Bransford, J. D., Brown, A. L., \& Cocking, R. R. (Eds.). Committee on Developments in the Science of Learning, Commission on Behavioral and Social Sciences and Education. Washington, DC: National Academy Press.

National Research Council. (2000). Educating teachers of science, mathematics, and technology: New practices for the new millennium. Washington, DC: National Academy Press.
National Research Council. (2001). Adding it up: Helping children learn mathematics. J. Kilpatrick, J. Swafford, \& B. Findell (Eds.). Mathematics Learning Study Committee, Center for Education, Division of Behavioral and Social Sciences and Education. Washington, DC: National Academy Press.

National Research Council. (2005, prepublication copy). Rising above the gathering storm: Energizing and employing America for a brighter economic future. Committee on Prospering in the Global Economy of the 21st Century: An Agenda for American Science and Technology. Washington, DC: National Academy Press.

National Science Board. (2004). Science and engineering indicators 2004. Arlington, VA: National Science Foundation.

Schmidt, W. H., McKnight, C. C., Houang, R. T., Wang, H. C., Wiley, D. E., Cogan, L. S., et al. (2001). Why schools matter: A cross-national comparison of curriculum and learning. San Francisco: Jossey-Bass.

Stone, C. N., Henig, J. R, Jones, B. D., \& Pierannunzi, C. (2001). Building civic capacity: The politics of reforming urban schools. Lawrence, KS: University Press of Kansas.

Supovitz, J. A., Mayer, D., \& Kahle, J. B. (2000). The longitudinal impact of inquiry-based professional development on teaching practice. Educational Policy, 14(3), 331-356.

Supovitz, J. A., \& Turner, H. M. (2000). The effects of professional development on science teaching practices and classroom culture. Journal of Research in Science Teaching, 37(9), 963-980.

Weiss, I. R., Pasley, J. D., Smith, P. S., Banilower, E. R., \& Heck, D. J. (2003). Looking inside the classroom: A study of K-12 mathematics and science education in the United States. Chapel Hill, NC: Horizon Research, Inc.

Wilson, S. M., \& Berne, J. (1999). Teacher learning and the acquisition of professional knowledge: An examination of research on contemporary professional development. Review of Research in Education, 24, 173-209.

Wiske, M. S. (Ed.). (1998). Teaching for understanding: Linking research with practice. San Francisco: Jossey-Bass.

Zhang, X., \& Wang, L. (2002). National Science Foundation local systemic change: Evaluation of data collection and analysis for cohort 1-4 projects on student achievement. Rockville, MD: Westat. 


\section{Nondiscrimination Statement}

The University of Pennsylvania values diversity and seeks talented students, faculty, and staff from diverse backgrounds. The University of Pennsylvania does not discriminate on the basis of race, sex, sexual orientation, religion, color, national or ethnic origin, age, disability, or status as a Vietnam era veteran or disabled veteran in the administration of educational policies, programs, or activities; admissions policies, scholarships, or loan awards; and athletic or University-administered programs or employment. Questions or complaints regarding this policy should be directed to Executive Director, Office of Affirmative Action, 1133 Blockley Hall, Philadelphia, PA 19104-6021 or 215-898-6993 (Voice) or 215-898-7803 (TDD).

\section{GRE Briefy}

Graduate School of Education

University of Pennsylvania

3440 Market Street, Suite 560

Philadelphia, PA 19104-3325

\section{About CPRE}

The Consortium for Policy Research in Education (CPRE) studies alternative approaches to education reform in order to determine how state and local policies can promote student learning. Currently, CPRE's work is focusing on accountability policies, efforts to build capacity at various levels within the education system, methods of allocating resources and compensating teachers, instructional improvement, finance, and student and teacher standards. The results of this research are shared with policymakers, educators, and other interested individuals and organizations in order to promote improvements in policy design and implementation.

CPRE unites five of the nation's leading research institutions to improve elementary and secondary education through research on policy, finance, school reform, and school governance. Members of CPRE are the University of Pennsylvania, Harvard University, Stanford University, the University of Michigan, and the University of Wisconsin-Madison.

CPRE Policy Briefs are published by CPRE. To learn more about CPRE research or publications, please call 215-573-0700 or access CPRE publications at www.cpre.org; www.wcer.wisc.edu/cpre/; or www.sii.soe.umich.edu.
NON PROFIT

U.S. POSTAGE

PAID

PERMIT NO. 2563

PHILADELPHIA, PA 